\title{
A New Race of Cercospora sojina in Hokkaido
}

\author{
Kayo ShIRAI*, Harukuni Horita**, Fumio TANAKA***, \\ Akio TANII*** and Yosuke Mino*
}

Key words : Cercospora sojina, soybean, race.

The frogeye spot disease of soybean caused by Cercospora sojina Hara has long been known in Hokkaido, Japan. However, few studies have been made on this disease until recently because its occurrence has been negligible and caused little damage to soybean. This disease occurred severely on a soybean variety, Suzuhime in Tokachi district in 1990. The formation of frogeye-like spots on the leaves is characteristic of this disease. The brown spots appear on the stems and pods at a later stage of growth, resulting in the formation of black seeds of low quality. On the other hand, the occurrence of this disease was first reported in the U.S.A., 1924 ${ }^{3}$. Since then this disease had prevailed in Midwest region till the end of the 1940's. However, it has recently become less severe due to the use of resistant variety. The five races are known to be involved in this disease in the U.S.A. ${ }^{1,2,4)}$ However, little is known about the races of this disease found in Hokkaido and the varietal difference of soybean to this disease.

The identification of race was carried out by the method of Phillips and Boerma ${ }^{2}$. The pathogens were isolated from a single spore on the infected seeds of Suzuhime grown in the same field in Ashoro, Hokkaido. Five isolates (SCS9001 SCS9004 by Dr. Tanii, and H8 by Dr. Kodama) were used for this experiment. Ten soybean varieties (standard varieties for this test in the U.S.A.) were used for this experiment. C. sojina was cultured on potato-dextrose agar for 3-4 weeks on which sterilized water was poured, followed by rubbing softly the surface to make spore suspension. After filtration through two layers of gauze, the concentration of spores was adjusted to $6 \times 10^{4} \mathrm{cells} / \mathrm{ml}$. A soybean plant at the stage of 2-3 compound leaves grown in a greenhouse was sprayed with $2.5 \mathrm{ml}$ of spore suspension. The plants were placed at $25^{\circ} \mathrm{C}$ for $72 \mathrm{hr}$ under $100 \%$ humidity and then transferred to a greenhouse. The reaction of the varieties was examined in terms of the number of spots formed on the leaves 2 weeks after spraying with the spore suspension.

The varieties forming no spots or a small number of tiny brown spots were regarded as resistant, while those forming large spots of 2-3 $\mathrm{mm}$ as susceptible (Table 1). Table 2 shows the comparison of the races between the U.S.A. and Japan. Soybean varieties, Lee and Hill were resistant, while the other varieties were susceptible to all five isolates in Hokkaido. Besides, these five isolates were the same in terms of the symptoms on the varieties tested. Accordingly, all the isolates tested were concluded to belong to the same race. Our race did not correspond to any of the races reported in the U.S.A. (Table 2). This suggests that our race is a new one. Therefore, this new race was designated as race $\mathrm{H}$.

The reaction of 70 varieties of practical use to these five isolates was examined. The experimental procedures were the same as mentioned above. Figure 1 shows the reaction of soybean varieties to these

* Department of Agroenvironmental Science, Obihiro University of Agriculture and Veterinary Medicine, Inada-cho, Obihiro, Hokkaido 080, Japan 帯広畜産大学

** Hokkaido Prefectural Plant Protection Office, Naganuma, Yubari-gun, Hokkaido 069-13, Japan＼cjkstart北海道病 害虫防除所

*** Hokkaido Prefectural Tokachi Agricultural Experiment Station, Memuro, Hokkaido 082, Japan 北海道立 十勝農業試験場

**** Hokkaido Prefectural Donan Agricultural Experiment Station, Ono, Hokkaido 041-12, Japan 北海道立道 南農業試験場 
Table 1. Relative number of spots on soybean varieties and reaction to the isolates in Hokkaido

\begin{tabular}{|c|c|c|c|c|c|c|c|c|c|c|}
\hline \multirow{3}{*}{$\begin{array}{c}\text { Soybean } \\
\text { variety }\end{array}$} & \multicolumn{10}{|c|}{ Isolate } \\
\hline & \multicolumn{2}{|c|}{ SCS9001 } & \multicolumn{2}{|c|}{ SCS9002 } & \multicolumn{2}{|c|}{ SCS9003 } & \multicolumn{2}{|c|}{ SCS9004 } & \multicolumn{2}{|c|}{$\mathrm{H} 8$} \\
\hline & a) & b) & a) & b) & a) & b) & a) & b) & a) & b) \\
\hline Hood & 40.0 & $\mathrm{~S}$ & 28.8 & $\mathrm{~S}$ & 15.2 & $\mathrm{~S}$ & 65.2 & $\mathrm{~S}$ & 31.6 & $\mathrm{~S}$ \\
\hline Roanoke & 95.7 & $\mathrm{~S}$ & 55.0 & $\mathrm{~S}$ & 136.6 & $\mathrm{~S}$ & 59.0 & $\mathrm{~S}$ & 16.6 & $\mathrm{~S}$ \\
\hline Lee & 1.0 & $\mathrm{R}$ & 0.5 & $\mathrm{R}$ & 0.3 & $\mathrm{R}$ & 0.0 & $\mathrm{R}$ & 0.2 & $\mathrm{R}$ \\
\hline Hill & 0.0 & $\mathrm{R}$ & 0.0 & $\mathrm{R}$ & 0.0 & $\mathrm{R}$ & 0.0 & $\mathrm{R}$ & 0.0 & $\mathrm{R}$ \\
\hline Blackhawk & 123.3 & $\mathrm{~S}$ & 39.2 & $\mathrm{~S}$ & 20.6 & $\mathrm{~S}$ & 35.2 & $\mathrm{~S}$ & 15.8 & $\mathrm{~S}$ \\
\hline Kanrich & 67.7 & $\mathrm{~S}$ & 20.7 & $\mathrm{~S}$ & 52.8 & $\mathrm{~S}$ & 35.0 & $\mathrm{~S}$ & 16.0 & $\mathrm{~S}$ \\
\hline Davis & 65.5 & $\mathrm{~S}$ & 49.8 & $\mathrm{~S}$ & 72.2 & $\mathrm{~S}$ & 13.5 & $\mathrm{~S}$ & 15.5 & $\mathrm{~S}$ \\
\hline Bragg & 77.5 & $\mathrm{~S}$ & 39.0 & $\mathrm{~S}$ & 5.0 & $\mathrm{~S}$ & 89.3 & $\mathrm{~S}$ & 16.7 & $\mathrm{~S}$ \\
\hline Lincoln & 93.3 & $\mathrm{~S}$ & 24.0 & $\mathrm{~S}$ & 12.2 & $\mathrm{~S}$ & 32.2 & $\mathrm{~S}$ & 19.4 & $\mathrm{~S}$ \\
\hline Kent & 72.5 & $\mathrm{~S}$ & 11.0 & $\mathrm{~S}$ & 40.0 & $\mathrm{~S}$ & 49.3 & $\mathrm{~S}$ & 30.8 & $\mathrm{~S}$ \\
\hline Suzuhime & 25.4 & $\mathrm{~S}$ & 7.5 & $\mathrm{~S}$ & 8.3 & $\mathrm{~S}$ & 20.5 & $\mathrm{~S}$ & 9.2 & $\mathrm{~S}$ \\
\hline
\end{tabular}

a) Average number of spots/plant.

b) Reaction of variety, $\mathrm{S}$ : susceptible, $\mathrm{R}$ : resistant.

Table 2. Reaction ${ }^{a}$ of differential soybean varieties to the races of Cercospora sojina

\begin{tabular}{|c|c|c|c|c|c|c|}
\hline \multirow{2}{*}{ Soybean variety } & \multirow{2}{*}{$\begin{array}{c}\text { A new race }(\text { race } H) \\
\text { in Hokkaido }\end{array}$} & \multicolumn{5}{|c|}{ Race in U.S.A. ${ }^{\text {b) }}$} \\
\hline & & 1 & 2 & 3 & 4 & 5 \\
\hline Hood & $\mathrm{S}$ & $\mathrm{R}$ & $\mathrm{R}$ & $\mathrm{R}$ & $\mathrm{S}$ & $\mathrm{S}$ \\
\hline Roanoke & $\mathrm{S}$ & $\dot{\mathrm{R}}$ & $\mathrm{R}$ & $\mathrm{R}$ & $\mathrm{S}$ & $\mathrm{S}$ \\
\hline Lee & $\mathrm{R}$ & $\mathrm{R}$ & $\mathrm{R}$ & $\mathrm{S}$ & $\mathrm{S}$ & $\mathrm{R}$ \\
\hline Hill & $\mathrm{R}$ & $\mathrm{R}$ & $\mathrm{R}$ & $\mathrm{S}$ & $\mathrm{S}$ & $\mathrm{R}$ \\
\hline Blackhawk & $\mathrm{S}$ & $\mathrm{S}$ & $\mathrm{S}$ & $\mathrm{S}$ & $\mathrm{S}$ & $\mathrm{S}$ \\
\hline Kanrich & $\mathrm{S}$ & $\mathrm{R}$ & $\mathrm{R}$ & $\mathrm{R}$ & $\mathrm{R}$ & $\mathrm{R}$ \\
\hline Davis & $\mathrm{S}$ & $\mathrm{R}$ & $\mathrm{R}$ & $\mathrm{R}$ & $\mathrm{R}$ & $\mathrm{R}$ \\
\hline Bragg & $\mathrm{S}$ & $\mathrm{R}$ & $\mathrm{R}$ & R-S & - & $\mathrm{S}$ \\
\hline Lincoln & $\mathrm{S}$ & $\mathrm{R}$ & $\mathrm{S}$ & $\mathrm{R}$ & - & $\mathrm{R}$ \\
\hline Kent & $\mathrm{S}$. & $\mathrm{R}$ & R-S & $\mathrm{R}$ & - & $\mathrm{R}$ \\
\hline
\end{tabular}

a) $\mathrm{S}$ : susceptible, $\mathrm{R}$ : resistant, - : not tested.

b) Reported by Athow et al. ${ }^{1)}$, Ross ${ }^{4}$, and Phillips and Boerma ${ }^{2)}$.

isolates. The number of spots was expressed as the average of those formed by the five isolates, respectively. Great differences in the reaction were found among soybean varieties. Large and fused spots were generally observed on the leaves of the varieties which formed the great number of spots. Hon-iku 65 and Shirosengoku formed innumerable spots but their spots were tiny in size. Therefore these two varieties were regarded as resistant. Suzuhime was the most susceptible among the varieties tested. Koganejiro and PI.84751, parents of Suzuhime ${ }^{5}$, also formed large frogeye-like spots. Accordingly the present result supports that this disease occurred severely on Suzuhime in 1990. The varieties such as Chashouryu, Yuhime and Kariyutaka which do not form any spots will be of use for breeding of resistant varieties.

We gratefully acknowledge Dr. F. Kodama, Hokkaido Prefectural Kitami Agricultural Experiment Station for kindly supplying us his culture, Mr. H. Torikura, Hokkaido Prefectural Tokachi Agricultural Experiment Station for his valuable advice and National Institute of Agrobiological Resources, Hokkaido Prefectural Plant Genetic Resources Center and Soybean breeding laboratory of Hokkaido Prefectural Tokachi Agricultural Experiment Station for kindly supplying us their soybean cultivars. 


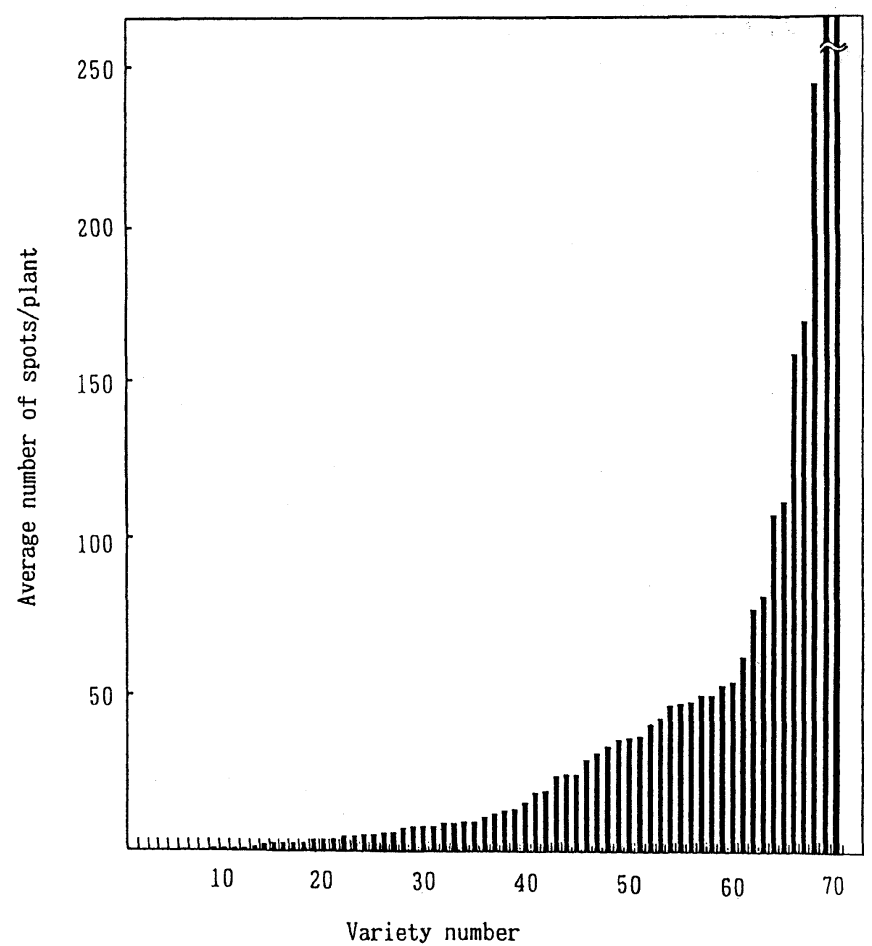

Fig. 1. Average number of spots on soybean varieties to 5 isolates (SCS9001, SCS9002, SCS9003, SCS9004, $\mathrm{H} 8)$

Variety

1 Chashouryu

2 Yuhime

3 Kariyutaka

4 Nagahadaka 1

5 Ishikarishiro 1

6 Otofuke-ousode

7 Okuhara 1

8 Miharudaizu

9 Shizunaidaizu

10 Chuseihadaka

11 Tokachihadaka

12 Rankoshi 1

13 Wasemidori

14 Shirobana-dairyuhadaka

15 Gokuwase-chisima

16 Tokachinagaha

17 Wasehadaka

18 Hokkaihadaka

19 Tokachishiro

20 Kitaminagaha

21 Karafuto (Toyohara)

22 Hosoba 1

23 Sakamoto-wase

24 Kitamishiro

25 Horoizumidaizu
26 Karafuto 1
27 Oyachi 2
28 Isuzu
29 Kitamusume
30 Bansei-hikarikuro
31 Marukotsubu
32 Shinsei
33 Chusei-hikarikuro
34 Suzunari
35 Nagahajiro
36 Nukanaidaizu
37 Tenpoku-shirome
38 Wasekogane
39 Yoshioka-tairyu
40 Toyomusume
41 Rankoshi
42 Karikachi
43 Shimoshirazu 1
44 Tokachikuro
45 Kanro
46 Tenpokuwase
47 Honkurakake
48 Tsurunoko (Odate)

\author{
49 Shiratsurunoko \\ 50 Hourai \\ 51 Himeyutaka \\ 52 Tsurukogane \\ 53 Asamidori \\ 54 Oshima-shirome \\ 55 Kitakomachi \\ 56 Wase-tsurunoko \\ 57 Toyokomachi \\ 58 Toyosuzu \\ 59 Tsurunoko (Kariwano) \\ 60 Fukunagaha \\ 61 Suzumaru \\ 62 Kitahomare \\ 63 Komamusume \\ 64 Shirokotsubu \\ 65 Yuuzuru \\ 66 Koganejiro \\ 67 Suzuhime \\ 68 PI.84751 \\ 69 Hon-iku 65 \\ 70 Shirosengoku
}




\section{Literature cited}

1. Athow, K.L., Probst, A.H., Kurtzman, C.P. and Laviolette, F.A. (1962). A newly identified physiological race of Cercospora sojina on soybean. Phytopathology 52: 712-714.

2. Phillips, D.V. and Boerma, H.R. (1981). Cercospora sojina race $5:$ A threat to soybeans in the southeastern United States. Phytopathology $71: 334-336$.

3. Phillips, D.V. and Boerma, H.R. (1982). Two genes for resistance to race 5 of Cercospora sojina in soybeans. Phytopathology $72: 764-766$.

4. Ross, J.P. (1968). Additional physiological races of Cercospora sojina on soybeans in North Carolina. Phytopathology 58 : 708-709.

5. Sunada, K., Sakai, S., Gotoh, K., Sanbuichi, T., Tuchiya, T. and Kamiya, M. (1981). A new soybean variety "Suzu-hime”. Bull. Hokkaido Prefec. Agric. Exp. Stn. 45 : 89-100.

\section{和 文 摘 要}

白井佳代・堀田治邦・田中文夫・谷井昭夫・美濃羊輔：北海道におけるダイズ斑点病菌, Cercospora sojina の新レー ス

1990 年に北海道十勝地方で, ダイズ斑点病が, 品種スズヒメに特異的に多発生した。そこで, この罹病スズヒメ種子 より 5 菌株を分離し，レース判別試験を行った。試験は, Phillips \& Boerma（1981）の方法に準じた。その結果, 5 菌株はすべて同一レースであり，これは，米国で報告されている既知の 5 レースとは異なる新レースであると考えら れた。したがって, 本レースをレース $\mathrm{H}$ と命名した。また, 北海道の実用品種において, 同様に接種試験を行ったと ころ, 葉の病斑数には品種間差がみられた。スズヒメは本病に対する感受性が最も高い品種のひとつであった。また, ユウヒメ，茶小粒，カリユタカなどは，まったく発病せず，抵抗性品種と考えられた。

(Received April 11, 1994) 